\title{
NURSE PRESCRIBING BY CHILDREN'S NURSES: VIEWS OF DOCTORS AND CLINICAL LEADS IN ONE SPECIALIST CHILDREN'S HOSPITAL
}

Molly Courtenay, BSc MSc PhD, Cert. Ed., Professor of Prescribing and Medicines

Management, University of Reading. Email: M.Courtenay@reading.ac.uk.

Nicola Carey, BSc MPH, Senior Research Fellow, University of Reading,

UK. Email: n.j.carey@ reading.ac.uk

Short Title: Doctors and clinical leads views of nurse prescribing

Address for correspondence: School of Health and Social Care, University of Reading, Bulmershe Campus, Reading, RG6 1HY. UK.

Email:M.Courtenay@Reading.ac.uk

\section{Acknowledgements}

We would like to acknowledge the funders of this study; North Central London SHA, and all the participants who gave their time generously. 


\section{ABSTRACT}

\section{Aim}

To explore the views of doctors and clinical leads, who care for children and young people, on nurse prescribing in one specialist children's hospital.

\section{Background}

Over 14, 000 nurses in the United Kingdom have virtually the same prescribing rights as doctors. Benefits of nurses adopting this role have been reported but doctors do have some concerns. Increasing numbers of specialist nurses involved in the care of children in the hospital setting are undertaking prescribing training. No research has explored the views of health care professionals on nurse prescribing in this setting. This is important as peer support can hamper prescribing.

\section{Method}

Interviews were conducted between October 2006 and July 2007 with 11 doctors and 4 clinical leads in one specialist children's hospital. This was a qualitative study and a thematic analysis was conducted on the interview data.

\section{Findings}

Participants reported that nurse prescribing improved access to medicines and continuity of care. Concerns included the need for doctors to have confidence in the ability of the nurse who wanted to become a prescriber, the selection of nurses for prescribing training, the effects of nurse prescribing on the individual roles and responsibilities of doctors, and nurses' clinical skills.

\section{Conclusion}


Doctors and clinical leads working in a specialist children's hospital in the United Kingdom recognise that nurse prescribing makes a positive contribution to the delivery of services to children and young people. However, it is important that nurses have the appropriate clinical skills and doctors understand nurse prescribing. This will only take place if there is good communication across professional boundaries.

\section{Relevance to Clinical Practice}

Selection processes for the prescribing programmes must ensure that students have the necessary course pre-requisites. Communication across professional boundaries is crucial to the successful implementation of nurse prescribing in the care of children and young people in the hospital setting.

\section{Keywords}

Nurse prescribing, hospital doctors, children and young people, qualitative approach 


\section{INTRODUCTION}

Until recently the roles and responsibilities of doctors in society were well understood (Royal College of Physicians 2005). Traditionally, doctors have predominantly been responsible for diagnosing and treating the illnesses of those who consult them for help (Freidson 1988). However, over the last 2 decades, these roles and responsibilities have been reshaped by social and political factors which have changed the expectation of what a medical role entails. This in turn has affected practice. The European Working Time Directive (i.e. legislative changes in 2004 which restricted the work hours of junior doctors in the National Health Service and other healthcare systems throughout Europe), is one such factor. Doctors themselves have different expectations of their role. The increasing proportion of women in the medical profession, who work part-time, and want a flexible working environment in order to meet the needs of their family, means that they contribute fewer hours to medicine than men. These restraints on doctor's availability mean they have been increasingly unable to meet patient demand (Rosen \& Dewar 2004).

Additionally, in order to meet the increasing expectation among the general public for timely, convenient access to an ever-wider range of health care services (Rosen \& Dewar 2004), a central aim of modern health care policy in the United Kingdom (UK) is to provide services which are both flexible and accessible to patients (Department of Health (DoH) 1999, DoH 2000). There has been increasing diversification of the roles of healthcare professionals (Rosen \& Dewar 2004). Consequently, over recent years, there has been growing emphasis on the expansion of nursing roles (DoH 1999). Nurses are 
increasingly taking up positions such as clinical nurse specialist and nurse consultant (Barnsteiner et al. 2002, Brady \& Neal 2000, Gibson et al. 2003). These roles frequently involve the prescription of medicines (Courtenay \& Carey 2008) once a practice restricted to doctors.

The potential benefits of the adoption of this role by nurses includes improved patient care without compromising patient safety, greater choice and increased access to medicines for patients, and improved use of healthcare professionals time and skills (DoH 2006).

Nurse Independent Prescribers (NIP) can assess, diagnose and prescribe independently any licensed medicine (including some controlled drugs (CDs)) provided that it is within their area of competence (DoH 2005). In contrast, supplementary prescribing, best suited to patients with chronic or long term conditions, takes place after assessment and diagnosis of a patient's condition by a doctor, and a Clinical Management Plan (CMP) has been drawn up for the patient. The CMP, agreed by the patient, nurse supplementary prescriber and doctor, includes a list of medicines (within the Nurse Supplementary Prescribers (NSP) area of competence) from which the supplementary prescriber is able to prescribe. NSP are able to prescribe any medicine (including CDs and unlicensed medicines). Following successful completion of the programme, nurses are awarded the dual qualification of NIP/NSP. There are now over 13,500 nurses across the UK qualified as both NIP and NSP (Nursing and Midwifery Council (NMC) 2007). 
Although a small number of studies have explored the views of healthcare professionals (primarily General Practitioners (GPs)) about nurse prescribing, little or no evidence is available that has explored the views of health care professionals in the hospital setting.

\section{BACKGROUND}

A small number of studies have examined the views of doctors on nurses adopting the role of prescribing (Avery et al. 2004, Courtenay \& Berry 2007, Latter et al. 2005). Perceived benefits reported by Avery et al. (2004) in telephone interviews with 6 hospital doctors and 6 GPs in one region in the UK include improved efficiency, reduced waiting times and more detailed information about medicines for patients. Doctors felt confident in the nurse's ability and level of knowledge, and most reported that they felt the prescribing programme did fulfil nurses' training needs.

More recently, benefits have been reported by GPs in a study by Courtenay \& Berry (2007). These researchers surveyed 30 GPs and 31 nurse prescribers to assess their views on what they believed were the main advantages and disadvantages of nurse prescribing. In addition to affirming the benefits reported by Avery et al. (2004), a perceived advantage for patients, reported by doctors, was continuity of care. However, disadvantages reported by these participants included reduced contact with doctors, threat to doctor's role, blurring of boundaries and nurse's limited skill base.

Similar findings were reported by Latter et al. (2005) who adopted a survey approach and in-depth evaluation of ten case study sites throughout England to evaluate independent 
extended nurse prescribing. Predominantly based in primary care, the 12 doctors interviewed at the 10 case study sites were supportive of nurses, who worked in their practice, adopting the prescribing role and reported that these nurses had sufficient experience to prescribe medicines. However, they did have some reservations about the larger population of nurses adopting this role. They also highlighted the importance of not underestimating the clinical skills required for the prescribing. Although participants reported that the medical profession felt threatened by nurse prescribing, they did however acknowledge that the prescription of medicines by nurses would, in the future, be seen as standard practice.

The importance of nurses' clinical skills has also been highlighted by Stenner et al (2008) reporting on interview findings from doctors and non prescribing nurses working in dermatology. Although participants were positive about the benefits experienced of nurse prescribing, the acceptance of nurse prescribing in general was reported as being conditional upon the selection of nurses with appropriate experience and training.

Concerns about nurse prescribers limited clinical skill base and competence have been noted in the medical press (Day 2005, Avery \& James 2007, McGavock 2007) and it is evident that the medical profession has expressed similar concerns about nurses who adopted other advanced nursing roles (Tye \& Ross 2000, Llyod Jones 2005). Adopting a case study approach to explore the role of the emergency nurse practitioner, a lack of understanding about the appropriateness of the nurse's clinical skills was reported by Tye 
\& Ross (2000) to explain the negative attitudes of the medical profession in one accident \& emergency department in the UK.

It is evident that although generally supportive, healthcare professionals have engaged in considerable debate about nurse prescribing and doctors do have some concerns about nurse's clinical skills and competence. However, very little research has examined the views of healthcare professionals in the hospital setting and no research has examined the views of these professionals on nurse prescribing in the care of children and young people. This is important given that an increasing number of specialist nurses working in secondary care, including those involved in the care of children and young people, are undertaking prescribing training, and a lack of peer support and objection by medical staff can act as a factor preventing or hampering nurse prescribing (Courtenay et al 2008).

\section{THE STUDY}

\section{Aim}

To explore the views of doctors and clinical leads (CLs), who care for children and young people, on nurse prescribing in one specialist children's hospital.

\section{Design}

This paper reports on a subset of qualitative data that formed part of a larger study exploring stakeholder views of nurse prescribing in the care of children and young people in one specialist children's hospital. 
The study adopted an intrinsic case study design (Stake 1994), undertaken purely because of the researcher's intrinsic interest, and need to obtain a better understanding of the case i.e. the specialist children's hospital. Interviews were used to capture the perspectives on nurse prescribing from a range of stakeholders as the richness and complexity of human experience would have been lost if questionnaires had been used to explore this relatively new area of practice. This paper reports on interview data from doctors and clinical leads. Further study findings, including interviews with qualified nurse prescribers, and patient questionnaires are reported elsewhere (? RCN Journal).

\section{Participants}

A purposive sample of doctors and clinical leads was taken to include doctors who worked alongside a nurse nurse prescriber $(n=7)$, doctors who acted as designated medical practitioners (DMPs) (i.e. responsible for the education and assessment of nurses during the prescribing course) $(n=4)$ and clinical leads $(n=4)$ who covered the clinical areas in which nurse prescribers worked. Of the 15 participants, $\underline{\mathbf{X} \text { worked in } ? \text { area of }}$ child health, $X$ worked in? and $x$ wirked in.$\underline{\text { DMPs were at registrar }(n=?) \text { and }}$ Consultant level (N=?) At the time of the study, the first group of nurses $(n=7)$ within the hospital, had just completed prescribing training.

\section{Data collection}

Data collection took place between October 2006 and July 2007. Although the researchers were completely independent of the Nursing Development Unit (NDU) within the hospital (and so had no previous relationship with participants), they did liaise 
with the Unit with regards to participants. Seven DMPs who supported and supervised the nurse prescribers throughout prescribing training programme, 7 physician colleagues and 4 clinical leads were identified and approached by the NDU and asked if they were willing to participate in the study. Interview dates were then arranged with a researcher from the University of Reading through an administrator in the NDU. Although all of those approached agreed to participate, interviews with three physician colleagues were not conducted as these staff were unavailable during dates arranged for interviews. The face to face interviews were held in mutually convenient locations within the hospital. Each interview lasted between 15-20 minutes and all participants gave permission for the interviews to be audio-taped and fully transcribed. All interviews were conducted by a researcher from the University of Reading. The researcher, who had a background in nursing, was an experienced interviewer with a wealth of experience in qualitative research methods.

The interview schedule was informed by a review of the relevant literature and national work in the area of nurse prescribing (Latter et al. 2005, Courtenay \& Carey 2008). Topics covered in the interview schedule included general views and experience of nurse prescribing, role changes resulting from nurse prescribing, support during the prescribing course, and effects on nurse/doctor relationship and practice.

\section{Ethical considerations}


A full research proposal was submitted for scrutiny by the Hospital Trust Research Ethics Committee and the University of Reading Ethics Committee. The study met the research governance criteria of these committees and approval to undertake the study was granted. All participants were initially approached by the NDU of the hospital and given an information sheet. Prior to the interview, participants had the opportunity to ask the researcher any questions, and were informed they could leave the study at any time. Participants were informed that all responses would be anonymised. Consent forms were signed by participants prior to interviews.

\section{DATA ANALYSIS}

A thematic analysis (involving familiarisation with the data, coding of transcripts, identification of categories and themes, defining themes, and the production of a written analysis of themes) was conducted (Braun \& Clark 2006). Interviews were transcribed from audio-tapes and the computer software programme 'ATLAS Ti' was used to aid coding and organising patterns across the data. Once coded, patterns in the data were identified and organised into themes until saturation of the data was achieved.

\section{Rigour}

The initial interviews were coded independently by two researchers who then worked together to clarify codes and agree on a coding frame for the remaining data. Member validation occurred in that a draft of the main findings was presented in a report to the specialist hospital and reviewed by those involved in the research. A high level of agreement and acknowledgement of the relevance of the findings were reported. 


\section{FINDINGS}

The analysis resulted in two main themes 'benefits' and 'concerns', relating to the views of doctors and clinical leads working in the specialist children's hospital. The two themes (each with 2 sub-themes) were very distinct and demonstrated that although doctors and clinical leads were able to recognise and identify a number of benefits from nurses who had adopted the prescribing role, they still had a number of reservations about its implementation in practice and its effect on roles and responsibilities.

\section{Benefits}

\section{Access and Efficiency}

The most immediately apparent benefit of nurse prescribing was the improved speed and flexibility of access to medication for patients. Doctors and CLs explained how improved access to medicines had reduced the amount of time patients had to wait at the hospital for their prescriptions, which ultimately improved the patient pathway.

" is of enormous benefit to the patients and in particular smoothing out the patient pathway" (CL3)

Participants reported that the process of delivering care had improved as medicines were administered in a more timely fashion:

"I certainly think having somebody who is on the ward who can prescribe there and then, not waiting for somebody to come back and write it up, has got to be a benefit" (CL1) 
"Having someone who can deal with getting the basic things that you are always going to need to do and getting that sorted does speed things up so it means that you're one step ahead you're in position to get on with $x$ if they need it. Rather than still be drawing up drugs and getting things ready. So it does help". (Dr7)

In addition to clinical responsibilities, many doctors had managerial responsibilities and held shared academic positions and were not on the hospital site every day. Participants believed that the increased availability of a nurse prescriber meant they were able to respond to patients needs more efficiently:

“Certainly it means when they [patients] come they don't have to wait as long because they might have to wait for me to come back from some management meeting or something like that". (Dr5)

\section{Continuity of care}

As well as improving access and efficiency, doctors and CLs reported that prescribing enabled nurses to improve the continuity of care patients received. This was seen as particularly important when dealing with children and their parents. This specialist hospital environment was described as having a high turn over of junior doctors who did not have the same opportunity as nurse prescribers to review patients on a regular basis. Nurses were thought to be better at building relationships with patients and their families, providing additional support, which resulted in a better exchange of information upon which to judge the most appropriate approach to treatment. Nurses were able to develop 
long-term relationships with patients as they were able to review their condition over a number of months and even years.

"because the junior doctors are here for a year, and are trying to cover everything.... Having somebody, who knows about growth hormones, who knows about $\mathrm{X}$, who knows about Y does give you that continuity" (CL1)

In addition to providing information this was seen as a positive way of encouraging parents and children to adhere to medication regimens. As a result, doctors felt that children and their parents received more information about medicines and their side effects, and developed a better understanding of their illness:

"I think the major advantages for the patients will be that they will get a much better in-depth discussion about the medication they are on, what the side effects are, and they will have a much better understanding of it because nurses are much better at doing the wider discussions and giving the patients and parents a bit more time than we do". (Dr4) 


\section{Concerns}

\section{Selection of nurses}

The selection of appropriate candidates for prescribing training was recognised by Drs and CLs as being important to the success of the initiative.

\footnotetext{
"It is a funny area here. Sometimes you suddenly hear that a nurse is doing some very involved course and you think, 'well, they're not quite ready for that yet', you know, it's not top of our priority list” (CL2).
}

It was important for doctors that they felt able to trust and have confidence in the ability of the nurse who wanted to become a prescriber. Consequently, they expressed some reservations about all nurses potentially becoming nurse prescribers. It was felt strongly that caution should be used in deciding which nurses should be put forward to undertake training to become a nurse prescriber. Participants reported that the nurses in the hospital who had undergone prescribing training had each worked in the same specialist area for between 10-20 years. Doctors therefore felt that they knew them very well, and with this came trust and confidence in their knowledge and ability to make effective clinical and prescribing decisions. All the nurse prescribers were viewed as experts in their clinical area by doctors and CLs and more knowledgeable than junior doctors:

"I think it's useful because a lot of the junior doctors obviously rotate through every three to six months and actually if anything someone who's a permanent team member is probably more familiar with the drugs and protocols and the dose ranges". (Dr7) 
"I have absolutely no reservations at all, in the context of my colleague, she is somebody who I have known for a number of years, and I trust implicitly, but I think you have got to have that relationship". (Dr2)

“From my own personal experience, I don't have any anxieties. I was supervising a very experienced nurse, who was planning to operate in a very limited area and it was very easy for me to be assured, that this person had adequate knowledge in order to be able to prescribe safely" (Dr5)

Doctors had some concerns about nurses who were already qualified nurse prescribers when they were appointed to the trust. In these instances, doctors were of the belief that these nurses should go through the same induction process as qualified medical prescribers entering the trust:

"This is the same situation as a doctor, I mean essentially if somebody undergoes training, which is properly structured and the training is evaluated in the way that most professional training is, then they should have the skill to do what that qualification qualifies them to do. Obviously there are always individuals, whatever their backgrounds, who have some reservations about the level of their skills. I don't see why that should be any different for a nurse, or for anybody else who prescribes".(Dr5) 
Some doctors and CLs felt the expectations of prescribing programme were unrealistic. Whilst the importance of developing competence in the area of physical assessment and diagnosis skills was recognised, it was felt that this was beyond the remit of the prescribing programme. Participants reported that several nurse prescribers were undertaking or planning to undertake additional training in the area of physical assessment and diagnosis, however, these nurses had only undertaken this training after qualifying as a nurse prescriber:

"It seems to me that there are certain vagaries about the course that we all struggle a little with. I think some of the expectations of the course were a little impractical. Senior and experienced nurses, had to very quickly learn a whole new set of skills for patient evaluation, they also had to have a really intensive course in physiology and pharmacology. In some cases what is actually required is more physiology and pharmacology than they had been expected to know in the past. So I think it was quite a tall order really". (Dr5)

\section{Clarifying roles and responsibility}

Whilst participants reported that doctors were generally supportive of nurse prescribing, there was a feeling that other doctors in the hospital were more reticent.

"Some of the doctors have to move into the $21^{\text {st }}$ century, and understand that this is going to happen and therefore they might as well be involved in it" (CL1) 
CLs reported that they felt that part of their role was to inform the medical staff of the potential benefits in the hope that eventually they would take it on board.

Participants reported underlying anxieties about how established roles and responsibilities had been affected by nurses adopting the prescribing role. Difficulties and concerns were expressed in relinquishing responsibility to nurses and losing track of patients in their caseload.

"Yes I think it might have to. It is almost at: "Well we will do it when we have to" mentality. I don't know that it is a fear of deskilling; it is a fear of a lack of or loosing power. It is: "I am the doctor; I am supposed to be in charge". Effectively you are giving some of those responsibilities to the nurse prescribers" (CL1)

Whilst some doctors strongly believed that what ever happened they were responsible for the patient, others spoke of their concern and confusion over the lines of responsibility and accountability within the team and hospital once a nurse became a prescriber:

"You know the doctor in charge of the patient might feel some sort of insecurity, because they can't actually keep an eye on what is going on”. (Dr4) 
“Again my anxiety is largely for the nurses involved; it doesn't seem at the moment clear, exactly what their responsibility is and if there is a mess up, who carries the can. I am not clear if a nurse prescriber prescribes something at a dreadfully wrong dose, and somebody is harmed as a result, who carries the can. Is that my MDU subscription or is it a separate thing? I think those areas are something that to me are not entirely clear". (Dr2)

"I think probably I haven't really transferred that responsibility in my own mind as yet. I still feel as if I am responsible”. (Dr2)

Interviewees reported that the medical profession in general may feel threatened by nurses adopting the prescribing role:

"I think a lot of doctors might be quite threatened by it and feel that prescribing has traditionally been one of their roles. I think we all recognise that nurses are very good at talking to patients, getting out of them what they are worried about and addressing that, which we [doctors] are not so good at, and so I think there will be those doctors who are worried about it [nurse prescribing] and those who will see it as beneficial". (Dr8)

Several participants felt that disagreements between nurse prescribers and doctors about clinical decisions could be a potential source of conflict: 
"If the team works well I cannot imagine huge problems. I suppose one could think about personality problems, possibly a nurse would prescribe something that the consultant in charge was not to happy with. If that sort of thing occurred, we should actually have a team policy and define what the grey areas are”. (Dr4)

Whilst this did not appear to have been a problem so far, one doctor commented on how effective communication might alleviate the problem. It was felt that the best way to reduce these anxieties was by ensuring everyone understood nurse prescribing and that a new set of boundaries and guidelines were established:

"Obviously with anything that is slightly new or slightly evolving in a different direction you may have people who will be anxious. I think the anxiety comes from them not understanding or not really realising what it has to offer. That is why it is important that you have all groups involved, and that you communicate, then it will work better". (Dr7)

\section{DISCUSSION}

This study is the first to specifically represent the views of hospital doctors and clinical leads on nurse prescribing in the care of children and young people. We acknowledge that the study is limited to our participants all of whom worked in very specialist areas of care, many of which are unique to the hospital where the study was conducted. Further research is required on the views of healthcare professionals on the role of prescribing by 
children's nurses working in the primary care setting. Additionally, further research is required to explore the views of patients (and parents) on the prescription of medicines by nurses caring for children and young people.

Our findings indicate that hospital doctors and CLs believe that there are number of benefits to be gained by nurses, who care for children and young people, adopting the role of prescribing. These benefits are comparable to those identified in other settings in which nurses prescribe medicines (Avery et al 2004, Latter et al. 2005, Courtenay \& Berry 2007).

Our findings also provide some insight into some of the concerns and anxieties doctors have about nurse prescribing for children and young people and the factors which contribute to these uncertainties and anxieties. These factors include role confusion, lack of knowledge of the prescribing role, selection of nurses for the prescribing programme, and a lack of confidence in prescribing training and preparation. However, our findings suggest that having an established relationship with the nurse, nurse's level of knowledge and expertise, effective communication between team members, establishing new boundaries and guidelines, and experience of tangible benefits to self and patients will each help to redress doctors' confidence about this role.

Nurses, caring for children and young people in the hospital under study worked at advanced levels and had responsibility for their own clinics. Consequently, prescribing served to support these existing structures and arrangements rather than being embraced 
as a means of exploring new ways of working. For example, as many of the doctors were not available for clinical duties every day, improvements to service delivery for children and young people, via the increased speed and access to medicines, were considered particularly important. There was also agreement that nurse prescribing supported continuity of care for these patients. These findings are in line with other research, in the primary care setting, that has indicated improvements in the timeliness of patient access to medicines (Courtenay \& Berry 2007, Latter et al. 2005). For example, in a study by Courtenay \& Berry (2007), improved access, and continuity of care were two of the perceived advantages for patients reported by GPs and nurse prescribers.

Several studies have reported that specialist nurses are more informative and knowledgeable than junior doctors (Avery et al 2004, 2005, Courtenay \& Berry 2007, Latter et al. 2005). Doctors in our study similarly reported that they believed that nurses were able to provide patients with more information about medicines and their side effects than doctors. In addition, participants in our study reported that nurse prescribers developed and maintained long term relationships with children and their families. Doctors believed that these long term relationships, combined with better medicines information, improved patients and carers understanding of their illness, which resulted in possible increased adherence to treatment regimens.

Concerns were expressed by participants about the selection processes for the prescribing programme and the need to ensure nurses, caring for children and young people, had the appropriate clinical skills for this training. Although not specifically in relation to 
children, these concerns have been highlighted previously (Latter et al. 2005, Avery 2004, Stenner et al 2008) and a necessary pre-requisite for candidates applying to undertake the prescribing programme, are that they have appropriate assessment and diagnostic skills (NMC 2006). A recent additional learning outcome stipulated by the NMC and specifically related to children, is that all prescribing students must be able to demonstrate that they are aware of their competencies if prescribing for a child, and, that they know when to refer (NMC 2008). Participants in our study reported that some nurses were undertaking clinical skills training following completion of the prescribing course. This highlights the need for funding organisations, managers and prescribing course leaders to ensure that nurses have undertaken training and attained the appropriate skills in the care of children and young people prior to undertaking prescribing training.

Participants in this study also emphasised that in order to trust and feel confident in the ability of the nurse prescriber with who they worked, it was essential to have a strong and well established relationship. Doctors in both Avery et al.'s (2004) and Latter et al. 's (2005) research who were supportive of nurse prescribing, similarly reported good preexisting professional relationships with nurse prescribers in their practice which provided a good foundation for this role.

In addition to confusion over roles and responsibilities, doctor's reluctance to relinquish control to nurses, and concerns over losing track of patients may have arisen from a lack of knowledge and understanding about nurse prescribing. Although not specifically exploring nurse prescribing, evidence of the difficulties that implementation of specialist 
and advanced nursing roles creates has previously been reported in the literature (Tye \& Ross 2000, Llyod-Jones 2005). For example, in a meta-analysis of 14 studies (LlyodJones 2005) it was reported that the most important factors to hinder the implementation of specialist and advanced nursing roles were role ambiguity and a lack of understanding of the nurse's role.

Inevitably cultural change within a profession and the implementation of new roles creates a loss of professional identity, and uncertainty (Handy 1999, Tye \& Ross 2000). In this study good communication within the team was seen as a way of establishing a new set of boundaries and addressing doctors concerns about their roles and responsibilities once a nurse had adopted the role of a prescriber. Team meetings, where clear goals, roles and responsibilities can be re-established are therefore one approach that could be adopted to help address these issues. During a period of change and readjustment, which naturally follows the introduction of a new initiative such as nurse prescribing, it is vital that managers provide support and guidance so that clear and specific roles and responsibilities can be redefined within the clinical team.

\section{CONCLUSION}

Doctors and CLs working in a specialist children's hospital in the UK are supportive of nurse prescribing and recognise that it makes a positive contribution to the delivery of services to children and young people. However, it is evident that doctors have a number of reservations about its implementation in practice and its effect on their roles and responsibilities. If nurse prescribing is to be successfully implemented in this setting, it is 
important that nurses have acquired the appropriate clinical skills prior to registering on the prescribing programme, and doctors understand nurse prescribing. This understanding will only take place if there is good communication across professional boundaries. 


\section{REFERENCES}

Avery, A., Savelyich, B.,Wright, L. (2004) Doctors' views on supervising nurse prescribers, Prescriber, 5, 56-61.

Avery, A. J.,James, V. (2007) Developing nurse prescribing in the UK, BMJ, 335, 316.

Barnsteiner, J. H., Richardson, V.,Wyatt, J. S. (2002) What do Pediatric Nurses Do? Results of the Role Delineation Study in Canada and the United States, Pediatric Nursing, 28, (2), 165169.

Brady, M. A.,Neal, J. (2000) Role Delineation Study of Pediatric Nurse Practitioners: A National Study of Practice Responsibilities and Trends in Role Functions, Journal of Pediatric Health Care, 14, 149-159.

Braun, V.,Clark, V. (2006) Using thematic analysis in psychology, Qualitative Research in Psychology, 3, 77-101.

Courtenay, M.,Berry, D. C. (2007) Comparing nurses' and doctors' views of nurse prescribing: a questionnaire survey, Nurse Prescribing, 5, (5), 1-6.

Courtenay, M.,Carey, N. (2008) Nurse Independent and Nurse Supplementary Prescribing Practice: National Questionnaire Survey, Journal of Advanced Nursing, 61, (3), 291-299.

Day, M. (2005) UK doctors protest at extension of nurse's prescribing powers, BMJ, 331, 1159.

DoH (1999) Saving Lives: Our Healthier Nation, Department of Health, London.

DoH (2000) The NHS Plan: A Plan for Investment, A plan for reform, Department of Health, London.

DoH (2002) Extending Independent Nurse Prescribing within the NHS in England: A guide for Implementation, Department of Health, London.

DoH (2003) Supplementary Prescribing for Nurses and Pharmacists within the NHS in England, Department of Health, London.

DoH (2005) Written Ministerial Statement on the expansion of independent nurse prescribing and introduction of pharmacists independent prescribing, $\mathrm{DoH}$, London.

DoH (2006) Medicines Matters A guide to the mechanism for the prescribing, supply and administration of medicines, DoH, London.

Freidson, E. (1988) Profession of Medicine: A study of the Sociology of Applied Knowledge, University Chicago Press Ltd, London.

Gibson, F., Kahir, K.,Pike, S. (2003) Nurse Prescribing: children's nurses' views, Paediatric Nursing, 15, (1), 20-25.

Handy, C. (1999) Understanding Organizations, Penguin Books, London.

Latter, S., Maben, J., Myall, M., Courtenay, M., Young, A.,Dunn, N. (2005) An evaluation of extended formulary independent nurse prescribing. Final Report, Policy Research Programme Department of Health \& University of Southampton

Lloyd Jones, M (2005). Role development and effective practice in specialist and advanced practice roles in acute hospital settings: systematic review and metasynthesis

Journal of Advanced Nursing 49 (2) 191-209 
McGavock, H. (2007) Inadequate nurse prescribing training will fuel adverse events, www.pulsetoday.co.uk, (8th March 2008)

NMC (2007) Number of Independent Extended Nurse Prescribers, Personal Communication with NMC August 2007.

Rosen, R.,Dewar, S. (2004) On Being a Doctor: Redefining medical professionalism for better patient care, London, Kings Fund Publications

Royal College of Physicians (2005) Doctors in Society: Medical Professionalism in a changing world, London, Royal College of Physicians

Snell, B. J. (1999) Issues Related to the Practice of Prescribing, JOGNN, 28, (5), 513519.

Stake, R. E. (1995) The art of case study research: perspectives on practice, Sage,

London.

Tye, C.,Ross, F. (2000) Blurring Boundaries: professional perspectives of the emergency nurse practitioner in a major accident and emergency department, Journal of Advanced Nursing, 31, (5), 1089-1096. 